\title{
How Do Regulated Nurse Professionals in Alberta Assess Geriatric Depression in Residential Care Facilities?*
}

\author{
Anna Azulai ${ }^{1}$ and Christine A. Walsh²
}

\begin{abstract}
RÉSUMÉ
Bien que la dépression gériatrique soit un problème de santé mentale répandu, grave et sous-évalué dans les établissements de soins pour personnes âgées, peu de recherches ont été réalisées sur ce thème au Canada. Cette étude exploratoire à méthodes mixtes examine les perspectives et les pratiques du personnel infirmier réglementé en matière d'évaluation de la dépression gériatrique dans les établissements de soins en Alberta. Les résultats d'enquêtes quantitatives $(n=635)$ et d'entrevues qualitatives $(n=14)$ indiquent que la dépression gériatrique n'est pas systématiquement évaluée dans ces milieux de soins en raison de multiples défis, incluant un protocole d'évaluation manquant de clarté, l'utilisation non uniforme de méthodes d'évaluation dans les établissements et leur utilité clinique contestée, la disponibilité limitée des professionnels en santé mentale dans ces établissements et les opinions divergentes concernant la responsabilité du personnel infirmier professionnel dans l'évaluation de la dépression. Les implications et les orientations futures de la recherche sont discutées.
\end{abstract}

\begin{abstract}
Although geriatric depression is a prevalent, serious, and under-recognized mental health condition in residential care facilities, there is a dearth of related research in Canada. This exploratory mixed methods study examines the perspectives and practices of regulated nurse professionals on assessment of geriatric depression in residential care facilities in Alberta. Findings from the quantitative surveys $(n=635)$ and qualitative interviews $(n=14)$ suggest that geriatric depression is not systematically assessed in these care settings due to multiple challenges, including confusing assessment protocol, inconsistent use and contested clinical utility of current assessment methods in facilities, limited availability of mental health professionals in facilities, and the varied views of regulated nurse professionals on who is responsible for depression assessment in facilities. Implications and future research directions are discussed.
\end{abstract}

1 MacEwan University, Edmonton, AB

2 University of Calgary, Calgary, $\mathrm{AB}$

* We thank Alberta Continuing Care Association (ACCA), Alberta Geriatric Nursing Association (AGNA), the College and Association of Registered Nurses of Alberta (CARNA), and the College of Licensed Practical Nurses of Alberta (CLPNA) for their assistance in data collection.

Manuscript received: / manuscrit reçu : 05/07/18

Manuscript accepted: / manuscrit accepté : 17/04/19

Mots-clés : vieillissement, santé mentale, détection, centres d'hébergement, assistance à l'autonomie

Keywords: aging, mental health, detection, nursing home, assisted living

La correspondance et les demandes de tirés-à-part doivent être adressées à : / Correspondence and requests for offprints should be sent to:

Dr. Anna Azulai

MacEwan University

School of Social Work

City Centre Campus

10700104 Avenue NW

Edmonton, AB T5J 4S2

(azulaia@macewan.ca) 


\section{Background}

Depression is the leading cause of disability in the world and a major contributor to the overall global burden of disease (World Health Organization [WHO], 2018). Depression in older adults, also termed geriatric depression, is a public health concern due to its high prevalence and negative implications for the well-being of older adults (WHO, 2017).

Geriatric depression comprises a constellation of affective, cognitive, and physiological manifestations as defined in the Diagnostic and Statistical Manual of Mental Disorders (DSM-5) (American Psychiatric Association, 2013). The diagnostic criteria for clinically significant depression state that five or more of the following nine symptoms must be present for at least two weeks: (a) depressed mood; (b) loss of interest or pleasure in activities; (c) changes in weight or appetite; (d) changes in sleep patterns; (e) psychomotor agitation or retardation; (f) low energy; (g) feelings of worthlessness; (h) poor concentration; and (i) recurrent suicidal ideation or suicide attempt (American Psychiatric Association, 2013). One of these symptoms must be depressed mood or loss of interest and pleasure in activities. Symptom count points to the severity level of depression. For example, mild depression is diagnosed when five to six aforementioned symptoms are present that are mild in severity (Zuckerbrot et al., 2007). In these cases, the person might experience only mild impairment in functioning. In contrast, depression might be deemed severe if a person experiences all nine of the depressive symptoms with severe impairment in functioning (Zuckerbrot et al., 2007). Moderate depression falls between these two categories.

In addition, older adults may experience depressive symptoms that do not fulfil the DSM-5 criteria for clinically significant depression (Heok \& Ho, 2008). These include individuals having a dysthymic disorder, subthreshold depression disorder, and depression due to dementia (Dillon et al., 2014). Zuckerbrot et al. (2007) pointed out that these less severe forms of depression should not be overlooked because older adults with these conditions are at risk for developing clinical depression in addition to facing multiple co-morbidities, high levels of stress, and social isolation without being diagnosed and treated (Hasche, Lee, Proctor, \& MorrowHowell, 2013).

Geriatric depression is especially prevalent and underrecognized in residential care facilities, such as nursing homes or long-term care facilities and assisted living settings (Canadian Institute for Health Information [CIHI], 2010; Davison et al., 2007; Evers et al., 2002; Seitz, Purandare, \& Conn, 2010; Snowdon, 2010; Maxwell, Gilbart, Wanless, \& Strain, 2011).
Despite the high prevalence of geriatric depression in residential care facilities, detection rates have been low (Bern-Klug, Kramer, \& Sharr, 2010; Snowdon, 2010; Watson, Zimmerman, Cohen, \& Dominik, 2009). Unrecognized and untreated geriatric depression leads to further health deterioration, increased medical care utilization, poor quality of life, and premature death (Boyle et al., 2004; Smalbrugge et al., 2006). Research in residential care facilities emphasises limited knowledge and education about the symptoms of geriatric depression (Jansen \& Murphy, 2009; Mitchel \& Kakkadasam, 2011; Molinari et al., 2008), limited availability of mental health specialists (Craig \& Pham, 2006; McKay, 2009; Molinari, Hedgecock, Branch, Brown, \& Hyer, 2009; Maxwell et al., 2011), presence of ageist and other misconceived beliefs (Choi, Wyllie, \& Ransom, 2009), and ineffective and unsystematic depression assessment methods and protocols (Davison et al., 2007; Heiser, 2004; McCabe, Davison, Mellor, \& George, 2008; Pachana et al., 2010; Soon \& Levine, 2002; Teresi, Abrams, Holmes, Ramirez, \& Eimicke, 2001; Wagenaar et al., 2003).

In Canada, about 44 per cent of the residents in these congregate care settings have been diagnosed with depression (CIHI, 2010). In addition, depression is common in residents with cognitive impairment due to dementias and other conditions (Fiske, Loebach Wetherell, \& Gatz, 2009; Thompson, Herrman, Rapoport, \& Lanctot, 2007). Depression prevalence in residential care facilities in Alberta ranges from 34 to 44 per cent (Maxwell et al., 2011).

There is a paucity of Canadian- and Alberta-specific research on how geriatric depression is recognized and assessed in facilities. Limited research, however, emphasises the importance of early recognition and effective assessment of geriatric depression in these care settings (Adams-Fryatt, 2010; CIHI, 2010; Conn et al., 2008; Maxwellet al., 2011).

\section{Assessment of Geriatric Depression in Residential Care Facilities}

Various standardised scales and non-standardised assessment strategies (e.g., observations, verbal reports, etc.) are available for depression, the most common of which are initial screening procedures and formal psychiatric diagnostic assessment (Conn et al., 2008). Initial screening procedures generally involve tests to identify persons at increased risk of health conditions (Grimes \& Schultz, 2002). Screening that generates positive results should always be followed by a full psychiatric diagnostic assessment (Pachana et al., 2010), conducted by a trained mental health professional in a standardised diagnostic interview based on psychiatric diagnostic criteria (American Psychiatric Association, 2013). 
Routine periodic screening for geriatric depression in residential care facilities has been identified as a means of increasing detection and timely referral to treatment (Azulai \& Walsh, 2015; Hasche et al., 2013; Huang et al., 2014; Soon \& Levine, 2002). However, data on the effectiveness and clinical utility of the growing number of depression screening tests in these care settings is mixed (Koopmans, Zuidema, Leontjevas, \& Gerritsen, 2010; Pachana et al., 2010). Currently, the only type of routine depression screening in residential care in Canada is embedded within the InterRAI Minimum Data Set resident assessment protocol (RAI-MDS 2.0) (Azulai \& Walsh, 2015), which is considered a powerful identification tool in many health domains (Saliba \& Buchanan, 2008). However, performance of the Depression Rating Scale (DRS), embedded in the RAI-MDS 2.0, has been questioned (Schnelle, Wood, Schnelle, \& Simmons, 2001; Shin \& Scherer, 2009).

National geriatric organizations in North America recommend that the DRS should always be supplemented with other validated scales for accuracy (American Geriatrics Society \& American Association for Geriatric Psychiatry [AGS \& AAGP], 2003; Canadian Coalition for Seniors' Mental Health [CCSMH], 2006). In the United States, the RAI-MDS 2.0 has been replaced by the newer RAI-MDS 3.0 as a mandated assessment protocol for American long-term care (LTC). Instead of the DRS, the RAI-MDS 3.0 includes the Mood Questionnaire, a self-report and observation version of the robust 9-item Patient Health Questionnaire (PHQ-9) (Saliba \& Buchanan, 2008). There is strong evidence for the superb performance of the Mood Questionnaire in residential care facilities for older adults in the United States (Saliba \& Buchanan, 2012). Azulai and Walsh (2015), in a systematic literature review of nine depression screening instruments in residential care facilities for older adults in the United States and Canada, recommend the use of the Mood Questionnaire (Saliba \& Buchanan, 2012) in the Canadian facilities.

Grimes and Schultz (2002) warn, however, that although screening can improve detection, it can also do harm if administered improperly, leading to either false positive or false negative results. Therefore, screening tests should be carefully selected by trained clinicians based on their psychometric performance, purpose of screening, residents' characteristics, and facility context (Pachana et al., 2010).

\section{Who Can Assess Residents for Geriatric Depression?}

As opposed to comprehensive diagnostic assessment measurements, depression screening scales may appropriately be used by a variety of disciplines
(CCSMH, 2006). However, as the accuracy of the screening is crucial for triggering appropriate treatment responses, the administrator should have knowledge not only of the psychometric properties of the screening measures and their administration procedures, but also of the distinction between screening and diagnostic assessment, as well as confidentiality regulations (CCSMH, 2006).

Physicians, nurse professionals, nursing assistants (termed health care aides [HCAs] in Canada), psychologists, and social workers have all been identified in the literature as suitable administrators of screening for geriatric depression in residential care facilities (AGS \& AAGP, 2003; CCSMH, 2006; Morgan, 2015). However, research demonstrates that residential care staff, regardless of the discipline, are not well-trained in recognizing and managing depression in old age (Bagley et al., 2000; Davison et al., 2007; Teresi et al., 2001) and often believe that depression is a natural consequence of aging and relocation to institutional living (Cepoiu et al., 2008; Davison, McCabe, Mellow, Karantzas, \& George, 2009; Hasche et al., 2013; McCabe et al., 2008; Ogbeide, \& Neumann, 2011).

There is no specific recommendation in the Canadian Clinical Best-Practice Guidelines on the Assessment of Depression in LTC as to who is responsible for depression screening (CCSMH, 2006). In this vacuum, the screening and assessment of depression in older adults is typically part of the well-being assessment in nursing practice (College and Association of Registered Nurses of Alberta [CARNA], 2019b; Registered Nurses Association of Ontario [RNAO], 2003). Indeed, some scholars suggest that direct care nurse professionals, in general, are well-positioned to perform depression screening because they interact with patients and should be able to observe symptoms on a daily basis (Clibbens \& Rylatt, 2008; Koopmans et al., 2010; Kurlowicz \& NICHE Faculty, 1997). Other studies however, found that nurse professionals experience considerable difficulty identifying depression in residents (Bagley et al., 2000; Kerber, Dyck, Culp, \& Buckwalter, 2008; Mitchell \& Kakkadasam, 2011; Teresi et al., 2001) due to a lack of familiarity with the residents (Kohler et al., 2005), ageist attitudes (Choi et al., 2009), and inadequate training of staff in geriatric mental health (Davison et al., 2007).

\section{Geriatric Residential Care Landscape in Alberta}

Statistics Canada (2011) has defined residential care facilities as congregate living settings with four or more beds that are funded, licensed, or approved by provincial or territorial departments of health or social 
services, regardless of the type of ownership. Facilities for seniors that do not provide any level of care are not included in this study.

In Alberta, residential care facilities for seniors are part of the continuing care system that includes short or long-term services for individuals with health conditions or disabilities (Alberta Continuing Care Association [ACCA], n.d). These services are typically provided in home care, facility living, and supportive living streams. Facility living in Alberta includes LTC or nursing homes under the Nursing Home Act (Province of Alberta, 2017), and auxiliary hospitals, under the Hospitals Act (Maxwell et al., 2011). LTC facilities are designed for persons with complex chronic care needs who are unable to remain in their homes and who require registered nurse (RN) services aroundthe-clock (ACCA, n.d.). On the other hand, supportive or assisted living settings are designed for residents without complex care needs, staffed by fewer personnel than in LTC, and combine accommodation services and other supports and care, including meals, housekeeping, and social activities. In supportive living, the level of care is usually defined from more independent to less independent, and services are contracted to private operators, including not-for-profit and for-profit providers.

Some supportive living facilities have designated beds, operated by the regional health authority Alberta Health Services, to accommodate residents with advanced health care needs and who can no longer manage in their own homes, even with the support of home care (Canadian Healthcare Association, 2009). These facilities are referred to as designated supportive living (DSL). In DSL, services include assistance with medications and with activities of daily living (ADL, i.e., personal hygiene, dressing, toilet use, locomotion, and eating), around-the-clock supervision by a licensed practical nurse (LPN), and access to an on-call RN seven days a week (Alberta Health Services, n.d.).

The study described in this article focused only on LTC and DSL settings because these types of facilities usually provide care for residents with similar levels of functioning, similar characteristics, and similar rates of depression (Maxwell et al., 2011). Home care was mentioned only when provided as a service in these facilities.

\section{Study Goal}

There is no research on the geriatric depression assessment practices of nurse professionals in Alberta and Canada. Due to the high prevalence of this condition in facilities in Alberta (Government of Alberta, 2015), it is important to investigate how geriatric depression is recognized and assessed in residential care in the province.
Hence, our goal for this exploratory study was to examine how regulated nurse professionals perceive, recognize, and assess geriatric depression in residential care facilities in Alberta and to answer the following research questions:

1. Who assesses older adults for depression in residential care facilities in Alberta?

2. What assessment methods do they use?

3. How often do they conduct assessments?

The study was approved by the institutional research ethics board at the University of Calgary (REB13-0058).

\section{Methods}

Study Design

The study employed a convergent, parallel mixed methods design (Creswell \& Plano-Clark, 2011), using a cross-sectional survey $(n=635)$ and follow-up qualitative semi-structured interviews $(n=14)$. This approach allowed for the triangulation of qualitative and quantitative research methods (Azulai \& Rankin, 2012) to collect rich data with the joint focus on depth and breadth of the study of this under-researched phenomenon.

\section{Quantitative Survey}

A self-report fact-finding quantitative survey questionnaire included 91 items in six sections, taking study participants about 15 minutes to complete. Section 1 inquired about facility characteristics (e.g., the type of facility, level of care, location, the number of beds, availability of mental-health services, etc.). Items in this section were assessed on either dichotomous (yes / no) or menu (check-all-that-apply) response formats.

Section 2 covered assessment practices, including methods and frequency of assessment with either dichotomous (yes/no) or menu response. Section 3 inquired about perceived successful practices versus barriers in depression assessment. Information on successful practices was collected by an open-ended question: "What facilitators or successful practices for identification and assessment of depression in residents can you share?" Barriers were measured using a 12-item Barriers to Working with Depressed Older People scale developed by McCabe, Davison, \& Mellor (n.d.), who reported good internal reliability of the scale (Cronbach's $\alpha=.84)$. Scale responses were measured on a 4-point Likert scale format ( $1=$ strongly disagree; 2 = somewhat disagree; 3 = somewhat agree; $4=$ strongly agree). Possible total scores on the scale ranged from 12 to 48 , with a higher score representing stronger perceptions of barriers. In addition, an open-ended item allowed for collecting information on barriers that could not have been accounted for in previous research: "What additional barriers, [not mentioned in the Barriers 
scale], to effective identification of residents with depression have you observed?".

Section 4 was devoted to clinical knowledge and beliefs about geriatric depression, measured by two different scales: The first was a 12-item Late-Life Depression Quiz (LLDQ), developed by Pratt, Wilson, Benthin, and Schmall (1992), who reported high internal consistency of the scale $(\alpha=.85)$ using the Kuder-Richardson formula (KR-20). LLDQ offered a "true," "false", or "don't know" response format, with a total score ranging from 1 to 12 . Higher scores indicated greater clinical knowledge of geriatric depression. The second scale was an 18-item Knowledge of Geriatric Depression Scale, which was a modification of the 14-item scale Views about Depression in Older People by McCabe et al. (n.d.).

The original scale measured three aspects of knowledge of depression: symptoms of depression, myths of depression, and responses to depression. Items were designed specifically for residential care, based on the DSM-IV-TR criteria for major depressive disorder (Davison et al., 2009; McCabe et al., 2008). Responses were measured on a 4-point Likert scale $(1=$ strongly agree, 2 = somewhat agree, $3=$ somewhat disagree, and $4=$ strongly disagree). The internal reliability of the original scale was low (Cronbach's $\alpha=.40$ ), although McCabe et al. (n.d.) commented that low internal reliability was expected as the items deliberately varied in their degree of difficulty. We added four knowledge items to the original scale to reflect the most recent findings in the scholarly literature on geriatric depression. These items were measured using the same 4-point Likert scale and included the following: "Changes in appetite and weight might be symptoms of depression", "Suicide attempts are not common in older people in residential care facilities", "Depression is common among older adults with dementia", and "Psychotherapy combined with anti-depressant medication is effective in treating older people with depression". Total scores on the resultant 18-item Knowledge of Geriatric Depression Scale ranged from 18 to 72, with higher scores indicating a better level of knowledge.

Section 5 covered depression-specific education, including type and length of training in depression, as well as familiarity with best-practice clinical guidelines for the assessment of geriatric depression in residential care facilities. Items were measured by either dichotomous (yes/no) or menu response scale.

Finally, section 6 collected personal information on respondents (e.g., occupation, education level, experience in working with older adults and residential care, demographic data, etc.). The section also included an item inquiring whether a participant is interested in an individual interview related to the survey topic. The online study survey was piloted in October 2013 with regulated nurse professionals in different types of residential care facilities in Calgary and Edmonton $(n=6)$, generating univocal positive reviews.

\section{Qualitative Interviews}

Due to the large geographical dispersion of participants, qualitative interviews were collected through face-to-face meetings, Skype videoconferencing, and telephone communication. The interview guide for semi-structured interviews comprised 31 questions that corresponded to specific sections in the quantitative survey to allow for subsequent cross-comparison of the findings. For example, questions such as "How do you usually recognize residents with depression in your facility" and "What assessment tools (e.g., screening scales and other means of depression detection) do you use in your facility" corresponded to the survey items in section 2 on assessment practices. Similarly, questions such as "What is your opinion on how normal depression is in old age" and "In your opinion, how common is depression in residents with dementia" corresponded to some items in the section 4 of the survey on knowledge and beliefs about geriatric depression. Finally, questions such as "What successful strategies in the process of identification and assessment of residents with depression in your facility would you share with other clinicians" and "What potential or actual barriers to the effective recognition and assessment of depression in residents would you identify in your facility" corresponded to section 3 of the survey on barriers and facilitators for depression assessment in facilities.

\section{Data Analyses}

In this study, the qualitative and quantitative data were collected and analysed independently and then compared. We exported quantitative data from the SurveyMonkey (2015) to the IBM SPSS 23 software for statistical analyses. Some of the item responses in the survey were missing. Calculations were conducted on available data without missing-data manipulation. Statistical procedures included descriptive and inferential methods. Inferential statistics included independent-samples $t$-test, one-way ANOVA, and chi square.

We analysed open-ended survey questions and qualitative semi-structured interviews using qualitative content analysis technique (Sandelowski, 2000). We audiotaped interviews and transcribed them verbatim before the data analysis. Member check procedure (Jick, 1979) allowed for increasing the trustworthiness of the qualitative content analysis findings by inviting 
participants' feedback to enhance the credibility of the data analysis. Seven out of 14 participants responded, providing positive appraisal.

We conducted the comparison between the findings using the integrative data analytic approach (Castro, Kellison, Boyd, \& Kopak, 2010), including the data transformation approach and data consolidation matrix (Jang, McDougall, Pollon, Herbert, \& Russell, 2008). For example, we quantified qualitative data from the open-ended survey on the barriers and facilitators in the depression assessment process to facilitate comparison of the major themes and subthemes against survey data on the barriers. The data consolidation matrix involved creating a blended database around main study constructs (Bazeley, 2006) by combining the results from both qualitative and quantitative data to integrate blended data for further analysis. In this study, the main constructs in the consolidation matrix derived from the survey questions, qualitative themes and subthemes from interviews and open-ended survey questions, illuminated by relevant quotes and, when applicable, by related numeric values from the quantitative findings. Given that the findings were extensive, it was not feasible to include the consolidated matrix in this article. Instead, we present the integration of the findings as a narrative in the next section.

\section{Results}

Participants

We collected the survey data and semi-structured interviews between December 2013 and February 2015. Using a SurveyMonkey (2015) web-link collector service, we distributed the survey to about 7,000 regulated nurse professionals in Alberta, using a variety of venues, including the Alberta Continuing Care Association (ACCA), the Alberta Geriatric Nursing Association (AGNA), the College and Association of Registered Nurses of Alberta (CARNA), the College of Licensed Practical Nurses of Alberta (CLPNA), and the Education Research Centre (ERC). The total number of the survey responses from all venues, online and paperbased surveys, $n=696$, constituted an approximate 9 per cent response rate. Out of the 696, a total of $n=635$ surveys were included in this study: 19 surveys were eliminated for not meeting the study eligibility criteria; 39 were incomplete; and there were 3 outliers.

More than half of the participants were from LTC (Table 1). Also, more than half of the participants were employed in one of the two provincial census metropolitan areas of Calgary or Edmonton; the rest came from a variety of municipal districts and counties in Alberta. Almost half (47\%) of participants were from private facilities, and almost all of them $(97 \%)$ were located in the urban sector.
Table 1: Participating residential care facilities

\begin{tabular}{lrr}
\hline Residential Care Facility Characteristics & $\boldsymbol{n}$ & $\%$ \\
\hline Type of Facilities ( $n=635)^{\mathrm{a}}$ & & \\
Long-term care (LTC) & 348 & 55 \\
Designated supportive living (DSL) & 203 & 32 \\
DSL/ LTC & 84 & 13 \\
Type of Ownership $(n=627)^{b}$ & & \\
Private & 294 & 47 \\
Government & 131 & 21 \\
Not-for-profit & 164 & 26 \\
Don't know & 38 & 6 \\
Sector $(n=629)^{c}$ & & \\
Urban & 610 & 97 \\
Rural & 19 & 3 \\
\hline
\end{tabular}

Note. LTC $=$ Nursing homes or long-term care facilities. DSL = Designated supportive living settings. DSL/LTC = Combined facilities with designated supportive living and long-term care beds.

a $n=635$ (number of participants who responded to the question).

b $n=8$ participants omitted this question.

c $n=6$ participants omitted this question.

As shown in Table 2, almost two thirds $(60 \%)$ of the survey participants were LPNs. Most were experienced in working with older adults $(66 \%)$ and in residential care settings $(56 \%)$. Nearly half $(41 \%)$ of participants assumed a role that combined supervisory and direct care duties. The majority (58\%) worked day shifts only, and nearly half $(46 \%)$ were full-time employees. Only about one third of the participants had some post-secondary education degree. Most participants were between 18 and 45 years of age (55\%). Finally, the majority were women $(95 \%)$, Caucasian $(63 \%)$, and born in Canada (60\%).

All survey participants were invited for a follow-up qualitative interview. Interested participants contacted the lead researcher via email or telephone or provided their contact information in the survey. Interview participants consisted of those who directly contacted the researcher via email or telephone after viewing the study ad, as well as the survey participants who expressed their interest in the interview and provided their contact information. The researchers continued to recruit interview participants until the point of data saturation was reached, resulting in $n=14$ interviews.

All interview participants were women. Eleven nurse professionals were Caucasian; additionally, there was one Black Canadian, one Filipina, and one Chinese. Two participants were relatively new professionals (1 to 5 years of nursing experience), another two were mid-career professionals (6 to 8 years of nursing practice), and the rest of the sample were mature nurse professionals (more than 9 years of experience). In terms 
Table 2: Demographic and employment characteristics of the survey participants $(n=635)$

\begin{tabular}{|c|c|c|}
\hline Survey Participant Characteristics & $n$ & $\%$ \\
\hline \multicolumn{3}{|l|}{$\operatorname{Sex}(n=516)^{a}$} \\
\hline Male & 26 & 5 \\
\hline Female & 490 & 95 \\
\hline \multicolumn{3}{|l|}{ Age at time of survey (years) $(n=516)^{a}$} \\
\hline $18-25$ & 27 & 5 \\
\hline $26-35$ & 124 & 24 \\
\hline $36-45$ & 134 & 26 \\
\hline $46-55$ & 113 & 22 \\
\hline $56-65$ & 101 & 20 \\
\hline $66+$ & 17 & 3 \\
\hline \multicolumn{3}{|l|}{ Education $(n=518)^{b}$} \\
\hline No post-secondary degree & 333 & 64 \\
\hline Post-secondary degree & 185 & 36 \\
\hline \multicolumn{3}{|l|}{ Born in Canada $(n=517)^{c}$} \\
\hline Yes & 308 & 60 \\
\hline No & 209 & 40 \\
\hline \multicolumn{3}{|l|}{ Ethno-cultural background $(n=516)^{\mathrm{d}}$} \\
\hline Caucasian & 323 & 63 \\
\hline Southeast Asian & 80 & 16 \\
\hline Black & 40 & 8 \\
\hline South Asian & 28 & 5 \\
\hline Chinese & 18 & 4 \\
\hline \multicolumn{3}{|l|}{ Type of nurse professionals $(n=519)^{\mathrm{e}}$} \\
\hline RN & 206 & 40 \\
\hline LPN & 313 & 60 \\
\hline \multicolumn{3}{|l|}{ Professional roles $(n=519)^{f}$} \\
\hline Supervisor & 79 & 15 \\
\hline Direct care professional & 176 & 34 \\
\hline Combined supervisory/direct care position & 212 & 41 \\
\hline Other & 52 & 10 \\
\hline \multicolumn{3}{|l|}{ Length of work with older adults (years) $(n=514)^{\mathrm{g}}$} \\
\hline $0-2$ & 70 & 14 \\
\hline $3-5$ & 102 & 20 \\
\hline $6-8$ & 79 & 15 \\
\hline $9-11$ & 57 & 11 \\
\hline $12+$ & 206 & 40 \\
\hline \multicolumn{3}{|c|}{ Length of work in residential care facilities (years) $(n=517)^{\mathrm{h}}$} \\
\hline $0-2$ & 96 & 19 \\
\hline $3-5$ & 127 & 25 \\
\hline $6-8$ & 84 & 16 \\
\hline $9-11$ & 58 & 11 \\
\hline $12+$ & 152 & 29 \\
\hline \multicolumn{3}{|l|}{ Employment workload $(n=518)^{i}$} \\
\hline Full-time & 239 & 46 \\
\hline 1 day a week & 2 & 0.4 \\
\hline 2-3 days/week & 92 & 18 \\
\hline 4 days/week & 84 & 16 \\
\hline Varies & 101 & 19.6 \\
\hline \multicolumn{3}{|l|}{ Work shift $(n=515) i$} \\
\hline Day & 301 & 58 \\
\hline Afternoon/evening & 122 & 24 \\
\hline Night & 50 & 10 \\
\hline Varies & 42 & 8 \\
\hline
\end{tabular}

Continued
Table 2: Continued

Note. $\mathrm{RN}=$ registered nurse. $\mathrm{LPN}=$ licensed practical nurse.

a Number of participants who responded to this question. Missing responses $n=119$.

b Number of participants who responded to this question. Missing responses $n=117$.

c Number of participants who responded to this question. Missing responses $n=118$.

d Missing responses $n=116$.

e Missing responses $n=121$.

f Missing responses $n=118$.

g Missing responses $n=117$.

h Missing responses $n=120$.

of the geographical distribution of facilities, most were in urban settings, predominantly in the Calgary and Edmonton areas; one participant was from a rural LTC setting.

Integrating Findings from the Survey and the Interviews

All but four participants in the survey and all interview participants considered depression assessment as an important issue in residential care facilities. However, nurse professionals reported profound challenges in the timely recognition of depression in these care settings in Alberta, specifically referring to

1) limited availability of trained mental health professionals;

2) inconsistent use of standardized or other assessment methods across facilities; and

3) lack of clear depression assessment protocol.

These challenges are described below in detail.

\section{Limited Availability of Trained Mental Health}

Professionals

Survey participants reported that professional mental health assessment for depression in older adults in Alberta residential care facilities was primarily conducted by various external mental health consultants hired by the regional health authority (Alberta Health Services [AHS]). These included psychiatrists (mentioned by $40 \%$ of participants); geriatric mental health community team from the AHS (by $40 \%$ ); registered psychiatric nurses (RPN) (by 37\%); social workers (by $30 \%$ ); and psychologists (by 14\%).

Aside from the external professionals, almost 39 per cent of participants stated that they did not have access to any mental health professionals employed at on-site facilities. Additionally, 49 per cent mentioned access to on-site social workers; 18 per cent, to RPNs; 11 per cent, to psychiatrists; 4 per cent, to psychologists; and 0.2 per cent, to pastoral care. Survey participants did not select physicians and on-site RNs as mental health professionals. In line with this notion, interview participants reported that RNs in both DSL and LTC facilities 
had, indeed, minimal roles in the depression assessment process. Although they routinely conducted physical assessments of residents with suspected depression, on-site RNs assumed primarily a coordinating role in the depression assessment effort rather than in the assessment itself. Contrary to the aforementioned survey results, however, interview participants indicated that family physicians in LTC and AHS home care case managers in DSL (who are RNs hired by the AHS) were, in fact, responsible for mental health assessment, referral for mental health consultations, and the uptake of mental health recommendations.

There was no univocal agreement among participants on the responsibility of nursing for the assessment of depression in facilities. For instance, 13 per cent of the survey participants reported that their employers did not believe that detecting depression is part of the nursing role. This notion was stronger among interview participants, who also mentioned perceived limited depression-specific training of nurse professionals as an explanation. As one interview participant suggested, "It would be wonderful if [depression assessment] could fall onto a social worker or psychiatric nurse, or somebody who has a more specialized training."

Only one interview participant endorsed full responsibility of nurses for the assessment of depression in facilities:

I do believe that depression detection should be integral to the nursing care. I think that RNs and LPNs should do that ... I think it should be a major part of our job and education because [depression] is not recognized and detected.

\section{Inconsistent Use of Standardized Scales and Other} Assessment Methods across Facilities

Survey participants mentioned various depression assessment methods used in residential care facilities in Alberta (see Table 3).

The least prevalent methods across all types of facilities for assessing depression were laboratory tests, such as blood samples (14\%), and inter-professional conferences $(0.2 \%)$. Contrary to the survey results, interview participants endorsed inter-professional conferences, although their use was perceived as inconsistent across facilities. In DSL, for example, some participants noted having regular weekly inter-professional meetings, while others identified monthly nursing rounds with the geriatric mental health teams, and still others reported only annual family conferences. In LTC, inter-professional conferences were conducted based on the internal facility processes, and sometimes included bi-weekly staff debrief meetings and at other times only quarterly care conferences.
The most prevalent methods of depression assessment in the survey were observations of residents by staff (cited by $92 \%$ of survey participants) and reports by family and friends (74\% of survey participants). Staffinitiated one-to-one interviewing of residents was mentioned by 62 per cent of survey participants and resident-initiated reports were indicated by 43 per cent. In this vein, interview participants confirmed that they rarely rely on resident-initiated reports because the older generation may under-report depression as a result of either condition-related communication challenges or perceived stigma of depression. They found family-initiated reports or observations of staff members more reliable.

Contrary to these survey findings, however, interview participants suggested that one-to-one interactions of professional nursing staff with residents was limited, which hindered opportunities for the effective observation of residents. Indeed, only one participant reported consistently using one-to-one interviewing with residents for depression assessment, whereas the remainder outlined relying on other staff, such as social workers, housekeeping, and HCAs (health care aides), who seemed to have more opportunities for one-toone interactions with residents than did nurses. They particularly emphasised the important role of HCAs. Because of their limited contact with residents resulting from the shortage of professional staff, regulated nurse professionals suspected they could easily miss fluctuating signs in residents or fail to notice a pattern, while the HCAs, if observant and skillful, could spot the deviation from a resident's usual behaviour owing to their daily interaction.

Only 69 per cent of survey participants reported using standardised screening scales (Table 3). Twenty-one per cent of the survey participants were not aware of any depression screening scales used in their facilities, and 7 per cent claimed that no such scales were used at their workplace. Even in LTC, where the RAI-MDS is mandated, the use of the embedded Depression Rating Scale (DRS) was reported only by 74 per cent of participants from these care settings. Similarly, most interview participants reported inconsistent use of screening scales for depression.

When standardised screening scales were used, the DRS was the most common $(68 \%)$, followed by the original Geriatric Depression Scale (GDS) (13\%) or the GDS-15 (12\%) (Table 4). Interview findings support this notion: the DRS was perceived as accessible and less demanding.

In non-mandated DSL settings, the AHS home care case managers instead used the RAI-HC - a similar resident assessment protocol, developed for home care settings, which also includes the DRS. Interview 
Table 3: Depression assessment method by type of care setting $(n=365)$

\begin{tabular}{|c|c|c|c|c|}
\hline Type of Assessment Method & Total $n^{\mathbf{a}}(\%)^{\mathbf{b}}$ & $\operatorname{LTC} n(\%)$ & DSL $n(\%)$ & DSL/LTC $n(\%)$ \\
\hline Observations of resident's behaviour & $581(92)$ & $323(93)$ & $183(90)$ & $75(89)$ \\
\hline Family/friends reports & $471(75)$ & $263(76)$ & $149(73)$ & $59(70)$ \\
\hline Staff-initiated verbal questioning of residents & $366(58)$ & $206(59)$ & $108(53)$ & $52(62)$ \\
\hline Resident-initiated reports & $254(40)$ & $135(39)$ & $83(41)$ & $36(43)$ \\
\hline Laboratory tests & $90(14)$ & $55(16)$ & $22(11)$ & $13(16)$ \\
\hline Total & 635 & 348 & 203 & 84 \\
\hline
\end{tabular}

Note. Percentages do not add up to 100 because participants were able to choose more than one response option. LTC $=$ Nursing homes/Long-term care facilities (including auxiliary hospitals). DSL = Designated supportive living settings. DSL/LTC = Combined types of facilities with designated supportive and long-term care beds.

a Number of participants who responded to this survey question.

b Percentage of respondents who selected specific response option from the total number of participants in the category.

participants from DSL indicated that when the DRS had triggered depression, the AHS-HC case managers sometimes used the GDS to corroborate the findings. This was not a formal requirement, and demanded extra work, thus was rarely used. Site RNs or LPNs in DSL, on the other hand, were not required to administer any depression screening scales besides cognitive capacity tests.

In addition, some participants from LTC questioned the clinical utility of the DRS, embedded in the RAIMDS. As one interview participant stated, "For us, the MDS is only for funding. We do not use MDS for clinical work." Also, in some LTC facilities, RAI-MDS assessments were done by specially designated nursing staff (termed MDS nurses) who collected information from
RAI-MDS reports and other staff documentation in medical charts without direct contact with residents. Other participants stated that the accuracy of the DRS values was heavily dependent upon individual interpretations of the questions by administrating staff as well as by residents. As one participant explained:
MDS has specific definitions for the questions that they ask. How that's interpreted is subjective for the person answering the questions. So, one of our biggest problems [is] making sure it is coded prop- erly. And sometimes, you will try to ask what you think fits the scale so that you get the answer that you want.

Another interview participant also expressed concern about the scales' accuracy related to the perceived

Table 4: Standardized screening scales for depression in residential care facilities $(n=624)$

\begin{tabular}{lc}
\hline Name of Screening Scale & $\boldsymbol{n}(\%)^{a}$ \\
\hline DRS in RAI-MDS 2.0 & $427(68)$ \\
Geriatric Depression Scale (GDS-30; GDS-15, GDS-5, or other) & $181(29)$ \\
Cornell Scale for Depression in Dementia (CSDD) & $34(5)$ \\
Patient Health Questionnaire, 9-item (PHQ-9) & $25(4)$ \\
RAI-HC & $13(2)$ \\
Single item screens & 8 (1) \\
Patient-Health Questionnaire, 2-item (PHQ-2) & 6 (1) \\
Hamilton Depression Rating Scale (HAM-D) & 5 (1) \\
Beck Depression Inventory (BDI) & $4(1)$ \\
Behavior Symptoms Mapping Tool (BSMT) & $4(1)$ \\
Montgomery-Asberg Depression Rating Scale (MADRS) & 2 (0.3) \\
Centre for Epidemiologic Studies Depression Scale (CES-D) & $1(0.2)$ \\
Zung Depression Rating Scale (ZSDS or SDS) & $1(0.2)$ \\
\hline
\end{tabular}

Note. Missing responses $n=11$. DRS = Depression rating scale. RAI-MDS $2.0=$ Resident Assessment Minimum Data Set, version 2. RAI-HC = Resident Assessment Minimum Data Set, Home-Care version. $n=132(21 \%)$ of participants were unaware of any specific depression screening scale in their facilities; $n=41(7 \%)$ pointed to no scales used.

a Percentage of respondents who selected specific response option from the total number of participants who responded to the survey question $(n=624)$. Percentages do not add up to 100 because participants were able to choose more than one response option. 
stigma of mental health, as well as the level of comprehension of the items by the residents, which might impact the accuracy of their responses:

It might be connected to stigma, doing these tests, so clients will respond in a certain way. They might not tell me what they really think and feel. There might be people that will give me the impression that everything is normal, but what I see is something else ... it is incongruent, right? Or sometimes they don't understand a question ... I might ask the same question again and get a different answer ... so, comprehension, right?

Some interview participants also drew attention to the importance of matching the tools to characteristics of residents for better effectiveness, such as using proper scales to identify depression in residents with cognitive decline. As one participant explained:

I just think that the DRS score of three and over is not as effective for people with dementia, and it is frustrating ...You find a positive score because, maybe, they have pain every day or they have this [face] expression because they are on specific [medications].

In addition to the DRS and GDS, most interviewed LTC and DSL participants (contrary to only $1 \%$ of survey participants) mentioned using the Behavior and Symptom Mapping Tool (BSMT) that, although not depression-specific, was endorsed by the AHS for daily behavioural symptoms in residents. The geriatric mental health team and some physicians require the BSMT screening results when there is a reported need for professional mental health assessment. However, some interview participants questioned the clinical utility and accuracy of the BSMT because, as one participant noted, "to get the HCAs to complete BSMT and not just copy the person who [completed] it before has been a challenge."

Nevertheless, most interview participants endorsed the use of standardised screening scales. As one participant pointed out, "using a screening scale would at least trigger something we do not see [or] notice ... ". Furthermore, participants emphasised the importance of using a variety of information sources for the assessment of depression in addition to screening scales, including residents' personal and medical histories in the pre-admission summaries, admission assessment notes, and post-admission staff progress notes in the depression assessment process. These notes, however, have value only if complete and accurate, which, as some participants claimed, "was often a missing piece".

\section{Lack of Clear Depression Assessment Protocol}

Over half of the survey participants indicated a lack of standard procedures for depression assessment.
Similarly, interview participants indicated an overall difference in procedures between LTC and DSL settings. In DSL, site RNs would provide referrals only to the AHS home care case managers, who would then conduct depression assessment, referring residents to physician, psychiatrist, or the geriatric mental health team if needed and coordinating the process.

In LTC settings, site RNs would assume a coordinating role. After recognizing signs of depression in residents or receiving a report from staff, residents, or families, they would make a referral for a formal psychiatric assessment to a physician, who in turn would assess affected residents and either prescribe treatment or refer cases for specialist psychiatric consultation by the geriatric mental health team. According to the interview participants, physicians or psychiatrists inconsistently followed the geriatric mental health team's recommendations, treatment, or re-assessments.

Inconsistent frequency of standardized screening in facilities, when used, was another challenge, regardless of facility type. Most of the interview participants noted that, in order to be effective, depression assessment and screening should be done for all residents routinely and not only after spotting depression symptoms. However, survey results (Table 5) show that residents were most often screened on an as-needed basis $(69 \%)$ or, to a lesser extent, only upon admission to a facility (37\%) and quarterly thereafter (30\%). Quarterly assessments occurred disproportionally more often in LTC than in DSL settings (Table 5), a notion confirmed by interview participants, who reported that formal depression assessment was conducted by AHS home care case managers at admission and then only annually. Admittedly, most interview participants were not sure what kind of frequency would be sufficient. As one participant noted, "Even a three-month [interval] between the DRS screenings might be too long for the timely identification of depression ... You can't wait that long to help a person."

In addition to the inconsistent frequency of depression screening, most interview participants pointed to the need for clear written procedures regarding what to do when the screening results are positive. As one RN noted, "What matters for clinical care is not only the ... method [of] recognizing symptoms of depression but also the action that follows, which may assist or fail the assessment process."

In summary, participants believed that depression assessment should be universal, routine, and frequent for all residents and that the timeline, methods of assessment, referral, and follow-up procedures should be clearly outlined. The assessment process of geriatric depression in DSL and LTC facilities differed substantially in many regards, including who assumed the 
Table 5: Frequency of the routine periodic screening for depression by facility type $(n=627)$

\begin{tabular}{lcccc}
\hline Frequency & LTC $(\boldsymbol{n}=\mathbf{3 4 8})$ & DSL $(\boldsymbol{n}=\mathbf{2 0 3})$ & DSL/LTC $(\boldsymbol{n}=\mathbf{8 4})$ & Total Number of Participants (\%) \\
\hline I don't know & 4 & 4 & 0 & $8(1)$ \\
Upon admission & 142 & 59 & 32 & $233(37)$ \\
Weekly & 5 & 7 & 3 & $15(2)$ \\
Monthly & 25 & 9 & 3 & $37(6)$ \\
Quarterly & 154 & 17 & 17 & $188(30)$ \\
Semi-annually & 9 & 10 & 2 & $21(3)$ \\
Annually & 44 & 39 & 13 & $96(15)$ \\
As needed & 228 & 148 & 59 & $435(69)$ \\
Never & 18 & 12 & 8 & $38(6)$ \\
\hline
\end{tabular}

Note. a Percentage of participants who selected specific response option from the total number of respondents to the survey question $(n=627)$. Percentages do not add up to 100 because participants were able to choose more than one response option. Missing responses $n=8$. DSL $=$ Designated supportive living; LTC = Long-term care; DSL/LTC = Combined types of facilities with designated supportive and long-term care beds.

responsibility for the assessment, who provided the referrals to mental health professionals, frequency of screening, and the use of assessment methods.

\section{Discussion}

This exploratory mixed methods study provided information, previously unavailable, on how regulated nurse professionals recognize and assess geriatric depression in residential care facilities in Alberta. The findings suggest that, despite the efforts of regulated nursing staff, geriatric depression is not systematically recognized and assessed in these care settings due to multiple challenges. Most study participants viewed the current depression assessment process in facilities as confusing, characterized by the lack of routine assessment and inconsistent use of the assessment methods. Nurse professionals relied for the initial recognition of depression symptoms on HCAs, other staff, and families of the residents; also, for depression assessment they relied on the external mental health professionals, despite the noted inconsistent availability of those professionals. There was also some reported uncertainty about the responsibility of the nurse professionals for the assessment of geriatric depression in residential care: Some believed depression assessment should be an integral part of the nursing practice, whereas others suggested that regulated nurse professionals do not possess sufficient depression-specific and mental health education to effectively recognize and assess for depression. These findings will be discussed below in the context of previous research, study limitations, implications, and future research directions.

\section{Depression Screening Considerations}

Previous research suggests that universal routine periodic screening for geriatric depression in residential care facilities increases the odds of detecting this mental health condition, decreases suicidal ideations, and contributes to the timely referral to treatment (Azulai \& Walsh, 2015; Hasche et al., 2013; Huang et al., 2014; Molinari et al., 2013; Oyama \& Sakashita, 2016; Soon \& Levine, 2002). Findings from this study support the importance of screening that is universal, routine, and frequent enough to allow for prevention and early detection of geriatric depression. Although quarterly routine assessment for depression has been mandated in LTC facilities in Alberta through the RAIMDS 2.0 (Azulai, 2016; Azulai \& Walsh, 2015), evaluating whether quarterly frequency is indeed sufficient for the timely detection of geriatric depression may be necessary due to the long-standing prevalence of this mental health condition in these care settings. Moreover, residents of DSL settings are at a disadvantage in comparison to those in LTC because in DSL routine screening is completed only annually.

Frequency and universality of screening is not the only challenge. Findings of this study reveal how the most often used depression screening tool in both LTC and DSL - the DRS - may be of limited clinical utility. These observations are congruent with the mounting evidence of the DRS limitations (Azulai \& Walsh, 2015). In the United States, the use of the DRS in nursing homes was discontinued in 2010 for its limited specificity and clinical utility and replaced by a robust, well-studied Patient Health Questionnaire (PHQ-9) modified for those care settings. In Canada, however, the DRS continues to be used, for the most part, as the only depression screening instrument. Even though the Canadian Clinical Best-Practice Guidelines on the Assessment of Geriatric Depression in LTC (CCSMH, 2006; Conn, Gibson, \& McCabe, 2014) recommend that the DRS always be supplemented by an additional scale, such as the Geriatric Depression Scale, to ensure effective identification of depression, our findings show that the Geriatric Depression Scale is not required and not 
often used in residential care facilities in Alberta. This under-utilization of the Geriatric Depression Scale in residential care facilities is consistent with other research in these care settings (Hammond, 2004; Pachana et al., 2010).

In designated supportive living facilities in Alberta, the use of standardised screening is less common than in LTC, which supports previous research on depression assessment in assisted living facilities in North America (Beuscher \& Dietrich, 2016; Watson et al., 2009). This is because DSL settings are regulated differently than for LTC: the DSL operators are not required to routinely screen their residents for depression using any standardized scales. Even though the AHS-HC case managers, employed by the regional health authority, conduct annual depression screening of DSL residents, they still use the same DRS scale with all aforementioned limitations. Moreover, annual screening falls behind quarterly screening, recommended by the Canadian Clinical Best-Practice Guidelines (CCSMH, 2006). There is a need to make the standards of depression screening in DSL at least equivalent to those of regulated LTC.

\section{A Lack of Clarity in the Depression Assessment Protocol in Facilities}

Findings of this study show that regulated nurse professionals perceived the current guidelines for depression assessment in facilities as confusing, inconsistent, and lacking clear direction, hindering the ability of nurse professionals to timely assisting residents with suspected depression. According to Molinari et al. (2013), geriatric depression assessment should include standardized screening, as well as ongoing interprofessional collaborative efforts to observe any changes in residents' physical and emotional status, regular one-to-one interviewing of residents and their families, regular laboratory tests for depression markers, review of personal and medical histories of residents, and the collection of other collateral data to create a wholesome picture of resident baseline behaviour and any deviations. Congruent with this notion, findings from our study reveal that regulated nurse professionals appreciate the high value of one-to-one communication with residents; however, they also often find one-to-one interactions with all residents are not feasible due to the current work force limitations in Alberta facilities.

Molinari et al. (2013) stated, "the proper assessment of problems is the cornerstone of effective treatment" (p. 41). Boyle et al. (2004), however, argued that detecting and diagnosing depression should be done in the context of treatment availability. There is little point in screening and assessing without acting upon the findings. Furthermore, initiating treatment for depression should not be the finish line; rather, there should be ongoing follow-up and regular reassessment of residents with depression for treatment evaluation and relapse prevention (Boyle et al., 2004). Thus, depression assessment protocol should include periodic medication reviews and treatment plan revisions in accordance with changes in a resident's condition (Bern-Klug et al., 2010; Davison et al., 2009). The Canadian Clinical Best-Practice Guidelines for the Assessment of Depression in LTC (CCSMH, 2006; Conn et al., 2014) could serve as a guiding compass in developing a clear depression assessment protocol in residential care facilities in Alberta. Therefore, increasing awareness of facility operators and staff about these clinical best-practice guidelines and promoting their implementation is important.

\section{Access to Mental Health Professionals for Residents with Depression}

Findings of this study reveal that regulated nurse professionals in residential care facilities in Alberta believed that the majority of mental health professionals available for residents with depression are external consultants, including psychiatrists, psychiatric nurses, social workers, and the geriatric mental health teams from the AHS. Access to these professionals was inconsistent, however, and availability of on-site mental health professionals was perceived as limited. Social workers were most often mentioned as the on-site mental health professionals in resident settings.

Interestingly, RNs were not perceived as mental health professionals in this study. Regardless of the facility type, RNs primarily assumed a coordinating role, rather than completing the depression assessment per se. Participants relied heavily on the HCAs, LPNs, and other staff in facilities; on resident families, and, to a lesser degree, on residents' reports to recognize symptoms of depression and refer residents for mental health assessment if needed. However, if those signs were missed or unreported, it would have been unlikely for the site RNs to become aware of the resident's mental health condition. Nurse professionals in the study made a connection between their comfort to conduct depression assessment and their perceived level of knowledge of geriatric depression, indicating the need for enhanced education in depression symptomology, treatment options, and counselling skills.

It is thought-provoking that not all regulated nurse professionals in this study believed that the assessment of depression is a part of the nursing practice. This finding contradicts the general provisions of the nursing regulatory bodies in Alberta: that regulated nurse professionals should assume the assessment of physical and emotional well-being of their clients 
(CARNA, 2019a, 2019b; CLPNA, 2019). This finding is congruent, however, with Waterworth, Arroll, Raphael, Parson, and Gott (2015), who reported variations in the sense of responsibility among nurse professionals for the assessment of depression in institutionalised older adults in New Zealand. Most nurses in that study believed that talking with patients about their depression was neither their responsibility nor within their scope of practice.

The Canadian Clinical Best-Practice Guidelines on the assessment of depression in LTC do not endorse any specific discipline, including nursing, as being responsible for depression assessment (CCSMH, 2006; Conn et al., 2008, 2014). Perhaps there is a need to revise the guidelines to make an evidence-based recommendation on the best-suited discipline for the role of depression recognition and assessment. Importantly though, any designated discipline among those suitable for the assessment role (e.g., nurse professionals, social workers, psychologists, physicians, etc.) requires proper geriatric mental health and counselling education to be effective (Bagley et al., 2000; Clibbens \& Rylatt, 2008; Kerber et al., 2008; Mitchell \& Kakkadasam, 2011; Koopmans et al., 2010; Teresi et al. 2001).

The Nursing Homes Act of Alberta (Province of Alberta, 2017) does not include any reference to mental health staff education or mental health services for residents. At this point, RPNs are the only mental health professionals mentioned in the Act, albeit only in the definitions section of the law but not among the list of required nursing home staff or elsewhere in the document. This has created a vacuum in the field of depression assessment and mental health, in general, which has been filled at the discretion of facility operators, clinical supervisors, and individual staff members. Revising the Nursing Home Act to include mental health would be timely.

Canadian LTC facilities are governed by the provincial legislation that creates standards for care and institution-specific policies and practices, which should be enforced and evaluated periodically for compliance (Kontos, Miller, Mitchell, \& Cott, 2010). According to Kontos et al. (2010), institutional compliance was straightforward when provincial legislation provided clear directives; however, when the legislation was left to interpretation, compliance resulted at the discretion of facility operators. Is it possible that leaving the legislative vacuum to the regulatory interpretations and personal discretion of facility staff has contributed to the lack of clarity about professional responsibility for depression assessment among nurse professionals? There is a need for further research to answer this question.

\section{Limitations}

This exploratory mixed methods study has limitations. First, the self-report nature of the quantitative survey could have introduced sampling bias (Polit \& Beck, 2008). Indeed, RPNs, male nurse professionals, and participants from auxiliary hospitals and rural settings in Alberta were underrepresented in this study.

Second, the response rate was 9 per cent despite the use of a variety of recruitment venues. The response rate was calculated based on the estimated 7,000 nurse professionals to whom the recruitment invitations were sent. However, it is unknown how many nurse professionals from this total number actually received invitations, due to inaccurate email addresses. Thus, the actual response rate could be higher. Also, the retained number of survey responses $(n=635)$ met and exceeded the estimated minimum sample of $n=365$ for this population (Raosoft Inc., 2004; SurveyMonkey Inc., 2015).

Third, it was expected that merging qualitative and quantitative data would generate some divergent findings, a common occurrence in mixed methods research (Creswell \& Plano-Clark, 2011). In this study, divergent findings, as reported in the results section of this article, were treated as opportunities to provide important additional insights because differences in findings may well reflect different views about geriatric depression and which are common in society (Creswell \& Plano Clark, 2011; Olive, 2014).

It is also possible that incongruences between quantitative survey data and qualitative interviews suggest that self-enrolled interview participants were not representative of the survey participants. This suggestion, however, may be overreaching because, for the most part, responses of the survey and interview participants were aligned. Rather, it seems, some divergence in opinions may be methodologically driven: Interview participants had the time to provide extensive responses about the complex study phenomenon, generating additional data. As the study goal was to obtain both in-depth and broadly applied initial insights into the under-researched phenomenon, the comparison of the qualitative and quantitative data allowed for the richness of findings that could not have been achieved using any one method alone.

Despite its limitations, this exploratory study provided previously unavailable information on the recognition and assessment of geriatric depression in residential care facilities in Alberta and elsewhere in Canada and offered specific practice-based recommendations to inform policy, clinical practice, and research to enhance geriatric depression care. As the study sample is relatively small, generalizing findings 
to all nurse professionals in residential care facilities in Alberta is limited. However, the study does provide a snapshot from the perspectives of RNs and LPNs in the province on the recognition and assessment practices and barriers to depression detection in mostly urban LTC and DSL facilities in the province. It may not, however, reflect the matter of things in auxiliary hospitals that were underrepresented in this study, as well as in rural facilities.

As geriatric residential care varies across Canadian provinces, generalizing these study findings to the rest of Canada may be of limited applicability. Yet, the commonalities between findings in this study and previous research in the United States, as we mentioned earlier, may suggest that results may be relevant to other Canadian provinces because depression care challenges are not unique to residential care facilities in Alberta.

Further research is needed to validate the findings of this study in a larger sample. Also, investigating perspectives, clinical knowledge, and assessment practices of other professional staff in residential care facilities in Alberta may improve understanding of the roles of other disciplines in the inter-professional care of residents with depression. Specifically, the role of social workers in the recognition and assessment of geriatric depression in residential care facilities should be explored for the potential contribution of social work profession in the provision of mental health services in geriatric residential care.

\section{References}

Adams-Fryatt, A. (2010). Acknowledging, recognizing, and treating depression in elderly long-term care residents. Annals of Long-Term Care, 18(11), 30-32.

Alberta Continuing Care Association (ACCA). (n.d.). Continuing care in Alberta. Retrieved from https://www.abcca.ca/about-continuing-care

Alberta Health Services. (n.d.). Designated supportive living. Retrieved from https:/ /www.albertahealthservices.ca/ cc/Page15490.aspx

American Geriatrics Society (AGS) \& American Association for Geriatric Psychiatry (AAGP). (2003). Consensus statement on improving the quality of mental health care in U.S. nursing homes: Management of depression and behavioural symptoms associated with dementia. Journal of American Geriatric Society, 51(9), 1287-1298.

American Psychiatric Association. (2013). Diagnostic and statistical manual of mental disorders (5th ed.). Washington, DC: Author.

Azulai, A., \& Rankin, J. (2012). Triangulation in Canadian doctoral dissertations on aging. International Journal of Multiple Research Approaches, 6(2), 125-140. doi: 10.5172/ mra.2012.6.2.125
Azulai, A., \& Walsh, C. (2015). Screening for depression in residential care facilities: A systematic narrative review. Journal of Gerontological Social Work, 58, 20-45. doi: $10.1080 / 01634372.2014 .904469$

Azulai, A. (2016). Recognition and assessment of geriatric depression in residential care facilities in Alberta: A mixed methods study of perspectives and practices of regulated nursing staff [Doctoral dissertation]. Retrieved from http://hdl. handle.net/11023/3188

Bagley, H., Cordingley, L., Burns, A., Mozley, C. G., Sutcliff, C., Challis, D., \& Huxley, P. (2000). Recognition of depression by staff in nursing and residential homes. Journal of Clinical Nursing, 9(3), 445-450.

Bazeley, P. (2006). The contribution of computer software to integrating qualitative and quantitative data and analysis. Research in the Schools, 13(1), 64-74.

Bern-Klug, M., Kramer, C. W. O., \& Sharr, P. (2010). Depression screening in nursing homes: Involvement of social services departments. The American Journal of Geriatric Psychiatry, 18(3), 266-275.

Beuscher, L., \& Dietrich, M. (2016). Depression training in an assisted living facility: A pilot study. Journal of Psychosocial Nursing and Mental Health Services, 54(5), 25-31. doi: 10.3928/02793695-20160201-01

Boyle, V. L., Roychoudhury, C., Beniak, R., Cohn, L., Bayer, A., \& Katz, I. (2004). Recognition and management of depression in skilled-nursing and long-term care settings: Evolving targets for quality improvement. The American Journal of Geriatric Psychiatry, 12(3), 288-295.

Canadian Coalition for Seniors' Mental Health (CCSMH). (2006). National guidelines for seniors' mental health: The assessment and treatment of mental health issues in long-term care homes. Toronto, ON: Author. Available at https://ccsmh.ca/wp-content/uploads/2016/03/ NatlGuideline_LTC.pdf

Canadian Institute for Health Information (CIHI). (2010). Depression among seniors in residential care. Analysis in Brief (May). Retrieved from https://secure.cihi.ca/ estore/productFamily.htm?pf=PFC1432

Canadian Healthcare Association. (2009). New directions for facility-based long term care. Ottawa, ON: Author.

Castro, F. G., Kellison, J. G., Boyd, S. J., \& Kopak, A. (2010). A methodology for conducting integrative mixed methods research and data analyses. Journal of Mixed Methods Research, 4(4), 342-360. doi: 10.1177/1558689810382916

Cepoiu, M., McCusker, J., Cole, M. G., Sewitch, M., Belzile, E., \& Ciampi, A. (2008). Recognition of depression by non-psychiatric physicians - A systematic literature review and meta-analysis. Journal of General Internal Medicine, 23, 25-36.

Choi, N. G., Wyllie, R. J., \& Ransom, S. (2009). Risk factors and intervention programs for depression in nursing 
home residents: Nursing home staff interview findings. Journal of Gerontological Social Work, 52(7), 668-685. doi: $10.1080 / 01634370802609155$

Clibbens, R., \& Rylatt, P. (2008). The role of nurse in assessment, diagnosis and management of patients with affective disorders. In S. Curran \& J.P. Wattis (Eds.), Practical management of affective disorders with older people (pp. 173-193). Oxon, ENG: Radcliffe.

College and Association of Registered Nurses of Alberta (CARNA). (2019a). About: What is CARNA?. Retrieved from https: / /www.nurses.ab.ca/about

College and Association of Registered Nurses of Alberta (CARNA). (2019b). Older adults policy pillar. Retrieved from https://www.nurses.ab.ca/about/what-is-carna/ policy-priorities-and-research/older-adults-policy-pillar

College of Licensed Practical Nurses of Alberta (CLPNA). (2019). CLPNA vision, mission, mandate $\mathcal{E}$ strategic plan. Retrieved from https://www.clpna.com/about-clpna/ vision-mission/

Conn, D., Gibson, M., Feldman, S., Hirst, S., Leung, S., MacCourt, P., ... Mokry, J. (2008). Guidelines for the assessment and treatment of mental health issues in LTC: Focus on mood and behaviour symptoms. Canadian Nursing Home, 19, 24-35.

Conn, D., Gibson, M., \& McCabe, D. (2014). 2014 guideline update - The assessment and treatment of mental health issues in long term care homes: (Focus on mood and behaviour symptoms). Toronto, ON: Canadian Coalition for Seniors' Mental Health (CCSMH). Available at https:/ / ccsmh.ca/wp-content/uploads/2016/03/2014-ccsmhGuideline-Update-LTC.pdf

Craig, E., \& Pham, H. (2006). Consultation-liaison psychiatry services to nursing homes. Australasian Psychiatry, 14, $46-48$.

Creswell, J. W., \& Plano Clark, V. L. (2011). Designing and conducting mixed methods research (2nd ed.). Thousand Oaks, CA: Sage.

Davison, T. E., McCabe, M. P., Mellor, D., Ski, C., George, K., \& Moore, K. A. (2007). The prevalence and recognition of major depression among low-level aged care residents with and without cognitive impairment. Aging and Mental Health, 11, 82-88. doi: 10.1080/13607860600736109

Davison, T. E., McCabe, M. P., Mellor, D., Karantzas, G., \& George, K. (2009). Knowledge of late-life depression: An empirical investigation of aged care staff. Aging and Mental Health, 13(4), 577-586. doi: 10.1080/13607860902774428

Dillon, C., Tartaglini, M.F., Stefani, D., Salgado, P., Taragano, F. E., \& Allegri, R. F. (2014). Geriatric depression and its relation with cognitive impairment and dementia. Archives of Gerontology and Geriatrics, 59(2), 450-456. doi: 10.1016/j.archger.2014.04.006

Evers, M. M., Samuels, S. C., Lantz, M., Khan, K., Brickman, A. M., \& Marin, D. B. (2002). The prevalence, diagnosis and treatment of depression in dementia patients in chronic care facilities in the last six months of life. International Journal of Geriatric Psychiatry, 17(5), 464-472.

Fiske, A., Loebach Wetherell, J., \& Gatz, M. (2009). Depression in older adults. Annual Review of Clinical Psychology, 5, 363-389. doi: 10.1146/annurev.clinpsy.032408.153621

Government of Alberta. (2015). RAI-MDS 2.0 quality indicator interpretation guide. Edmonton, AB: Author. Retrieved from https:/ / open.alberta.ca/dataset/aa056894-c85f4526-a3da-25cd64795082/resource/05bfbf33-070c-49d7a4ce-1fc7857741cd/download/cc-cihi-rai-guide-2015.pdf

Grimes, D. A., \& Schulz, K. F. (2002). Uses and abuses of screening tests. The Lancet, 359(9309), 881-884.

Hammond, M. F. (2004). Doctors' and nurses' observations on the Geriatric Depression Rating Scale. Age and Ageing, 33(2), 189-192.

Hasche, L. K., Lee, M. J., Proctor, E. K., \& Morrow-Howell, N. (2013). Does identification of depression affect community long-term care services ordered for older adults? Social Work Research, 37(3), 255-262. doi: 10.1093/swr/svt020

Heiser, D. (2004). Depression identification in the longterm care setting. Clinical Gerontologist, 27(4), 3-18. doi: 10.1300/J018v27n04_02

Heok, K. E., \& Ho, R. (2008). The many faces of geriatric depression. Current Opinion in Psychiatry, 21(6), 540-545. doi: 10.1097/YCO.0b013e328311cdae

Huang, H., Chuang, Y., Hsueh, Y., Lin, P., Lee, B., \& Chen, C. (2014). Depression in older adults with stroke living in long-term care facilities. Journal of Nursing Research, 22(2), 111-118. doi: 10.1097/jnr.0000000000000028

Jang, E. E., McDougall, D. E., Pollon, D., Herbert, M., \& Russel, P. (2008). Integrative mixed methods data analytic strategies in research on school success in challenging circumstances. Journal of Mixed Methods Research, 2(3), 221-247. doi: 10.1177/1558689808315323

Jansen, I., \& Murphy, J. (2009). Residential long-term care in Canada: Our vision for better seniors' care. Ottawa, ON: Canadian Union of Public Employees (CUPE). Retrieved from https://archive.cupe.ca/updir/CUPE-long-termcare-seniors-care-vision.pdf

Jick, T. D. (1979). Mixing qualitative and quantitative methods: Triangulation in action. Administrative Science Quarterly, 24(4), 602-611.

Kerber, C. S., Dyck, M. J., Culp, K. R., \& Buckwalter, K. (2008). Antidepressant treatment of depression in rural nursing home residents. Issues in Mental Health Nursing, 29(9), 959-973. doi: 10.1080/01612840802274651

Kohler, M., Rabinovitz, T., Hirdes, J., Stones, M., Carpenter, G. I., Fries, B. E., ... Jones, R. N. (2005). Measuring depression in nursing home residents with the MDS and GDS: An observational psychometric study. BioMed Central Geriatrics, 5. doi: 1-1186/1471-2318-5-1 
Kontos, P. C., Miller, K.-L., Mitchell, G. J., \& Cott, C. A. (2010). Dementia care at the intersection of regulation and reflexivity: A critical realist perspective. The Journals of Gerontology: Series B, Psychological Sciences and Social Science, 66B, 119-28. doi: 10.1093/geronb/gbq022

Koopmans, R. T. C. M., Zuidema, S. U., Leontjevas, R., \& Gerritsen, D. L. (2010). Comprehensive assessment of depression and behavioral problems in long-term care. International Psychogeriatrics, 22(7), 1054-1062. doi: 10.1017/S1041610210000736

Kurlowicz, L. H., \& NICHE Faculty. (1997). Nursing standard of practice protocol: Depression in elderly patients. Geriatric Nursing, 18(5), 192-200.

McCabe, M. P., Davison, T., \& Mellor, D. (n.d.). A training program for professional careers in recognizing depression: Part I - Needs assessment. Melbourne, AUS: Deakin University.

McCabe, M. P., Davison, T. E., Mellor, D., \& George, K. (2008). Knowledge and skills of professional carers working with older adults with depression. Aging and Mental Health, 12(2), 228-235. doi: 10.1080/13607860701797166

McKay, J. R. (2009). Continuing care research: What we have learned and where we're going. Journal of Substance Abuse Treatment, 36(2), 131-145. doi: 10.1016/ j.jsat.2008.10.004

Mitchell, A. J., \& Kakkadasam, V. (2011). Ability of nurses to identify depression in primary care, secondary care and nursing homes: A meta-analysis of routine clinical accuracy. International Journal of Nursing Studies, 48(3), 359-368. doi: 10.1016/j.ijnurstu.2010.05.012

Molinari, V. A., Chiriboga, D. A., Branch, L. G., Greene, J., Schonfeld, L., Vongxaiburana, E., \& Hyer, K. (2013). Influence of mental health assessment on prescription of psychoactive medication among new nursing home residents. Clinical Gerontologist, 36, 33-45. doi: 10.1080/07317115.2012.731475

Molinari, V., Hedgecock, D., Branch, L., Brown, L. M., \& Hyer, K. (2009). Mental health services in nursing homes: A survey of nursing home administrative personnel. Aging \& Mental Health, 13(3), 477-486. doi: $10.1080 / 13607860802607280$

Molinari, V., Merritt, S., Mills, W., Chiriboga, D., Conboy, A., Hyer, K., \& Backer, M. A. (2008). Serious mental illness in Florida nursing homes: Need for training. Gerontology and Geriatrics Education, 29, 66-83.

Morgan, J. (2015). Palliative psychotherapy in the treatment of geriatric depression: A review of evidence-based psychogenic options. Innovative Issues and Approaches in Social Sciences, 8, 46-59. doi: 10.12959/issn.1855-0541. IIASS-2015-nol-art03

Ogbeide, S. A., \& Neumann, C. A. (2011). Knowledge of aging and late life depression among a sample of nonphysician clinicians: A preliminary analysis. American Journal of Psychological Research, 7, 19-30.
Olive, J. (2014). Reflecting on the tensions between emic and etic perspectives in life history research: Lessons learned. FQS Forum: Qualitative Social Research/Sozialforschung, 15(2), art. 6. Retrieved from http://www.qualitativeresearch.net/index.php/fqs/article/view/2072/3656

Oyama, H., \& Sakashita, T. (2016). Long-term effects of a screening intervention for depression on suicide rates among Japanese community-dwelling older adults. The American Journal of Geriatric Psychiatry, 24(4), 287-296. doi: 10.1016/j.jagp.2015.10.008

Pachana, N. A., Helmes, E., Byrne, G. J. A., Edelstein, B. A, Konnert, C. A., \& Pot, A. M. (2010). Screening for mental disorders in residential aged care facilities. International Psychogeriatrics, 22(7), 1107-1120. doi: 10.1017/ S1041610210000128

Polit, D. E., \& Beck, C. T. (2008). Nursing research: Generating and assessing evidence for nursing practice (8th ed.). Philadelphia, PA: Lippincott Williams \& Wilkins.

Pratt, C. C., Wilson, W., Benthin, A., \& Schmall, V. (1992). Alcohol problems and depression in later life: Development of two knowledge quizzes. The Gerontologist, 32(2), 175-183.

Province of Alberta. (2017). Nursing Homes Act: Nursing Homes Operation Regulation, Alberta Regulation 258/1985. With amendments up to and including Alberta Regulation 164/2015. Edmonton, AB: Alberta Queen's Printer. Retrieved from http:/ / www.qp.alberta.ca/documents / Regs/1985_258.pdf

Raosoft Inc. (2004). Sample size calculator. Retrieved from http:/ /www.raosoft.com/samplesize.html

Registered Nurses Association of Ontario (RNAO). (2003). Screening for delirium, dementia and depression in older adults. Toronto, ON: Author.

Saliba, D., \& Buchanan, J. (2008). Development and validation of a revised nursing home assessment tool: MDS 3.0. Santa Monica, CA: Rand Health.

Saliba, D., \& Buchanan, J. (2012). Making the investment count: Revision of the Minimum Data Set for nursing homes, MDS 3.0. Journal of American Directors Association, 13(7), 602-610.

Sandelowski, M. (2000). Whatever happened to qualitative description. Research in Nursing E Health, 23, 334-340.

Schnelle, J. F., Wood, S., Schnelle, E. R., \& Simmons, S. (2001). Measurement sensitivity and the MDS depression quality indicator. Gerontologist, 41(3), 401-405.

Seitz, D., Purandare, N., \& Conn, D. (2010). Prevalence of psychiatric disorders among older adults in long-term care homes: A systematic review. International Psychogeriatrics, 22(7), 1025-1039. doi: 10.1017/S1041610210000608

Shin, J. H., \& Scherer, Y. (2009). Advantages and disadvantages of using MDS data in nursing research. Journal of Gerontological Nursing, 35, 7-17. 
Smalbrugge, M., Jongenelis, L., Pot, A. M., Eefsting, J. A., Ribbe, M. W., \& Beekman, A. T. (2006). Incidence and outcome of depressive symptoms in nursing home patients in the Netherlands. The American Journal of Geriatric Psychiatry, 14(12), 1069-1076.

Snowdon, J. (2010). Depression in nursing homes. International Psychogeriatrics, 22(7), 1143-1148. doi: 10.1017/ S1041610210001602

Soon, J. A., \& Levine, M. (2002). Screening for depression in patients in long-term care facilities: A randomized controlled trial of physician response. Journal of American Geriatric Society, 50(6), 1092-1099.

Statistics Canada. (2011). Residential care facilities 2009/2010. Catalogue no. 83-237-X. Ottawa, ON: Author. Retrieved from https://www150.statcan.gc.ca/n1/pub/83-237x/83-237-x2012001-eng.pdf

Maxwell, C. J., Gilbart, E., Wanless, D., \& Strain, L. A. (2011). Designated assisted living (DAL) and long-term care (LTC) in Alberta: Selected highlights from the Alberta Continuing Care Epidemiological Studies (ACCES). Edmonton, ON: ACCES Research Group, University of Alberta. Retrieved from https: / / era.library.ualberta.ca/items /86c45141-a2bd49fc-a694-87c8ce185e4e

SurveyMonkey. (2015). Create surveys, get answers. Retrieved from https:/ / www.surveymonkey.com/

Teresi, J., Abrams, R., Holmes, D., Ramirez, M., \& Eimicke, J. (2001). Prevalence of depression and depression recognition in nursing homes. Social Psychiatry and Psychiatric Epidemiology, 36(12), 613-620.

Thompson, S., Herrmann, N., Rapoport, M. J., \& Lanctôt, K. L. (2007). Efficacy and safety of antidepressants for treatment of depression in Alzheimer's disease: A meta-analysis. The Canadian Journal of Psychiatry, 52(4), 248-255.

Wagenaar, D., Colenda, C. C., Kreft, M., Sawade, J., Gardiner, J., \& Poverejan, E. (2003). Treating depression in nursing homes: Practice guidelines in the real world. Journal of the American Osteopathic Association: Clinical Practice, 103(10), 465-469.

Waterworth, S., Arroll, B., Raphael, D., Parsons, J., \& Gott, M. (2015). A qualitative study of nurses' clinical experience in recognizing low mood and depression in older patients with multiple long-term conditions. Journal of Clinical Nursing, 24(17-18), 2562-2570. doi: 10.1111 /jocn.12863

Watson, L. C., Zimmerman, S., Cohen, L. W., \& Dominik, R. (2009). Practical depression screening in residential care/assisted living: Five methods compared with gold standard diagnosis. The American Journal of Geriatric Psychiatry, 17(7), 556-564.

World Health Organization (WHO). (2018). Depression. Retrieved from https://www.who.int/news-room/ fact-sheets/detail/depression

World Health Organization (WHO). (2017). Mental health in older adults. Retrieved from https://www.who.int/ news-room/fact-sheets/detail/mental-health-of-olderadults

Zuckerbrot, R. A., Cheung, A. H., Jensen, P. S., Stein, R. E. K., Laraque, D., \& the GLAD-PC Steering Group. (2007). Guidelines for adolescent depression in primary care (GLAD-PC): Identification, assessment, and initial management. Pediatrics, 120(5), e1299-e1312. doi: 10.1542/ peds.2007-1144 